\title{
Evaluation of Tuberculin Skin Test Response and Interfering Factors in Patients with Juvenile Idiopathic Arthritis
}

\author{
Işıl Eser Şimşek, Müferet Ergüven, Olcay Bilgiç Dağcı
}

Göztepe Teaching and Research Hospital, Istanbul, Turkey.

Email: olcaybilgic@yahoo.com

Received October $30^{\text {th }}, 2012$; revised November $30^{\text {th }}, 2012$; accepted December $8^{\text {th }}, 2012$

Copyright (C) 2013 Iş1 Eser Şimşek et al. This is an open access article distributed under the Creative Commons Attribution License, which permits unrestricted use, distribution, and reproduction in any medium, provided the original work is properly cited.

\begin{abstract}
Juvenile idiopathic arthritis (JIA) is the most common rheumatologic disease in pediatric age group. Mycobacterium tuberculosis infection (TB) is an important cause of mortality and morbidity in patients with inflammatory rheumatologic disease. The objective of this study is to determine to what extent active disease and use of drugs in JIA affects response to PPD skin test and thus to investigate the significance of PPD skin test in the diagnosis of latent TB. 77 children diagnosed with JIA according to ILAR diagnostic criteria and routinely followed by our rheumatology clinic were included in the patient group. Patients were grouped according to subtypes of disease, activity status and drugs they used. Control group was formed from 58 healthy children. PPD skin test was applied to each subject and the number of BCG scars of all cases was recorded. We found no significant difference in PPD induration diameters between JIA and control group ( $p>0.05)$. The number of BCG scar is similar in both groups. In the control group, age and number of BCG scars and PPD skin test diameter are positively correlated. But there is no such significant relationship in patients with JIA $(p>0.05)$. PPD induration diameter of patients with active disease is significantly shorter than patients in remission $(\mathrm{p}>0.05)$. PPD induration diameter of patients treated with steroid and disease modifying anti-rheumatic drug (DMARD) and underwent remission were not significantly different from the control group. When compared with patients using other drugs, patients on remission using steroid and DMARD have shorter PPD induration diameter. Activity of disease and drugs used (steroid, DMARD) affects PPD response. In the diagnosis of latent TB, normal range of PPD diameter in healthy child changes in JIA patient with active disease. That the PPD diameter is shorter than normal range could indicate underlying TB infection. This fact should be considered in the follow-up of the patients with JIA.
\end{abstract}

Keywords: PPD; Juvenile Idiopathic Arthritis

\section{Introduction}

Juvenile idiopathic arthritis (JIA) is the most common rheumatologic disease of childhood. In the treatment of acute period control of inflammation, prevention of deformities and protection of joint functions is targeted, while limitation of complications of disease and treatment, to provide normal growth and development, rehabilitation of patient and education of family is purposed in a long period. Extra-articular involvement is common in JIA $[1,2]$. Th1 cells are predominant in its pathogenesis. Mycobacterium tuberculosis infection commonly seen in patients with inflammatory rheumatoid disease is an important cause of mortality and morbidity. Though the diagnostic techniques of tuberculosis proceed everyday because of the fact that both these techniques are expensive and there is no consensus about the sensitivity, PPD is still important in the diagnosis of tuberculosis. PPD reaction is a typical delayed hypersensitivity reaction and its formation requires normal $\mathrm{T}$ cell functions. Since Th1 cells play an important role in JIA pathogenesis, JIA patients are expected to show higher PPD response with respect to healthy children. However, there are investigations on the suppression of delayed hypersensivity response in adults with rheumatoid arthritis (RA) [3], but there are no reports in the literature of similar studies in children with JIA. Immunosuppression due to disease or drugs used in the treatment of disease (steroid etc.) could interfere with the PPD reaction and cause difficulty in the 
detection of the infection.

In our study, we aimed to evaluate to what extent active disease and immunosuppressive drug use affect PPD response so to detect the role of PPD test in the diagnosis of latent tuberculosis.

\section{Material and Method}

\subsection{Study Group}

77 children (average age $11.9 \pm 4.33$ ) who are regularly followed in our rheumatology outpatient clinics because of JIA diagnosed according to ILAR criteria and 58 healthy children (average age $11.9 \pm 4.33$ ) followed by our healthy child outpatient clinic as a control group are taken into our study group. The children with JIA are categorized into three groups as remission without medication, remission with medication and active. There were 31 patients $(40.3 \%)$ in the remission without medication group, 39 patients $(50.6 \%)$ in the remission with medicateon and seven patients $(9.1 \%)$ with active disease. The patients in the remission group are the one who do not have any JIA related complaints in the last six months and whose CHAQ scores were less than 0.5. The active patients had JIA related complaints in the last six months and their CHAQ scores were more than 0.5. The patients with JIA are grouped into subtypes of this disease: Seven of them had systemic JIA, 34 had oligoarticular JIA, 26 had polyarticular JIA and 10 had enthesitis related arthritis.

Besides that, the patients were categorized according to the medication they were taking. 26 of JIA patients were taking DMARD (disease modifying anti-rheumatic drugs), 17 both DMARD and steroid and 2 anti-TNF. Tuberculin skin test with the Mantoux method were applied intradermally to all cases and their number of BCG scars were recorded.

For this study ethical approval is taken from Ethic Committee of Goztepe Teaching and Research Hospital.

\subsection{Tuberculin Skin Test}

Tuberculin Skin Test was made by applying 5TU PPD intradermal solution to the volar surface of the left front arm with a tuberculin injector. For the sake of standardization, these tests were applied and evaluated by the same doctor. The emergence of $6-10 \mathrm{~mm}$ papule around the injection area was accepted as the sign of correct application of the test. The longest diameter of the endurance around the injection area was measured and recorded after 72 hours with the pencil method.

\subsection{Statistical Analysis}

The results of this study were evaluated using NCSS
2007 \& PASS 2008 Statistical Software (Utah, USA). Since PPD measurements did not show normal distribution, Kruskall Wallis test was applied in the group comparisons besides descriptive statistical methods (average, standard deviation). In the detection of the differentiating group and evaluations according to two groups, Mann Whitney U test was used. In the PPD correlations, Spearman correlation analysis was utilized. In the quailtative data comparison, chi-square test was applied. The results were evaluated in the confidence range of $95 \%$ and significance $\mathrm{p}<0.05$ level.

\section{Results}

There was no significant difference in the age, gender and BMI of both groups. When PPD induration values of JIA and control groups were compared, no statistical significant difference was found $(\mathrm{p}>0.05)$. The number of BCG scars showed similar distribution in both groups. In the healthy group, positive correlation and significant relation $(p<0.05)$ were seen between PPD induration, age and BCG scars. In the JIA group, the effect of age and number of BCG scars on PPD was not found. Moreover, there was not found a significant relation between PPD values, JIA subtypes and follow-up period ( $p>$ $0.05)$. According to disease activity state, PPD induration values showed significant difference. The significance was due to the active group. PPD values of the active group were significantly lower than the ones of the remission patients. The PPD values of the patients in remission who had been treated with the combination of DMARD and methylprednisolone were lower than the ones treated with other medication. Furthermore, the PPD values of the patients in remission and active patients who take both DMARD and methylprednisolone didn't show a significant difference $(\mathrm{p}>0.05)$. The patients who take both DMARD and methylprednisolone even though they were in remission have lower PPD values similar to results of active patients.

\section{Discussion}

Tuberculosis with the incidence rate of $34 / 100,000$ in Turkey is a common disease [4]. It is recommended that BCG vaccines be made at the early age and screening be conducted with PPD tests on the healthy children in the societies with high tuberculosis incidence [5]. PPD reaction is a typical delayed hypersensitivity reaction and its formation depends on the normal $\mathrm{T}$ cell functions. The most important feature of JIA is inflammatory synovitis. In the diseases with the defected $T$ cell functions such as JIA, PPD reaction can be affected and it can cause low sensitivity in the detection of latent TB infection. Moreover, immunosuppressive treatment used in these patients 
creates an extra ambiguity in the PPD evaluation.

When the induration values of both JIA and healthy cases are compared in our study, a statistically significant difference was not detected. In the literature, a similar study was conducted to the rheumatoid arthritis cases by Wolfe et al. and the percent of PPD positivity was found as $9.2 \%$ [6]. When this rate is compared to the rates in the US population, it is found that PPD response didn't decrease in the rheumatoid arthritis patients. Many studies in the literature have shown that the infection rate increased in healthy children with the age [7,8]. Furthermore, the conducted studies found out that the vaccines in older ages caused larger indurations $[9,10]$. Many studies reported out that there is a positive correlation between PPD reaction and number of BCG scars [11-13]. In our study, similar to literature results PPD induration values went up in the control group as their age and number of BCG scars increased. On the contrary, the fact that PPD responses are not affected by these factors in patients with JIA can be caused by a possible defect in the late hypersensitivity reactions.

According to disease activity state (remission without medication, remission with medication and active), PPD induration diameter ratios show significant difference ( $p$ $<0.05)$. The significance was caused by the active group. PPD values of the active group were significantly lower than the ones of the remission patients $(p<0.05)$. There was no significant difference between PPD responses of patients in remission with medication and without medication. In the study of Waxman et al., rheumatoid arthritis patients were grouped into anergic and reactive categories according to their responses to the skin test with specific allergens. During these tests, it was found that there was no difference in disease activity between these two groups but anergic patients had longer active disease period [14].

The purpose in the comparison of medication used by patients in remission is to determine the effects of medication and activation on the immune system while keeping the effect of the disease. The average PPD induration diameters of the remission cases treated with the combination of DMARD and methylprednisolone have statistically lower significance than the cases treated only with DMARD. The purpose of the comparison of the patients in remission using medication to the active patients is to explore the effect of activation of the disease on the immune system. The PPD induration values of the patients in remission with medication who take DMARD and methylprednisolone and active patients who take the same medication didn't show significant difference. We can deduce from our study that disease activation affects PPD response and the cases in which methylprednisolone and DMARD were taken even though they are in remis- sion delayed hypersensitivity is affected as much as the effect of the disease activation.

In the literature, there are many publications with many different results which investigated the effects of immune suppressive medications especially methylprednisolone on the PPD response. In the Hoeyeraal's study where the patients who took $7.4 \mathrm{mg} /$ day of methylprednisolone were investigated, the PPD responses of the patients who took methylprednisolone were compared to the ones who did not take it. That study did not find any significant difference [15]. In the Hayes et al. study, similarly the same results were found out for the RA patients taking prednisone [16]. On the contrary to these results, Helliwell et al. compared the PPD responses of 50 RA and 50 healthy cases and found out that immune suppressive treatment was used more often for the RA patients with anergic [17]. In E. Kiray's study, it was determined that neither the activity of the disease nor the use of corticosteroid and methotrexate affected the PPD response [18]. In I. Sezer's study, the PPD responses of RA patients who were followed up without treatment were significantly higher than healthy cases and RA patients who were taking DMARD and methylprednisolone. In addition to this exploration of that study, it detected that the factors which are responsible of pathogenesis of the disease suppressed the late hypersensitivity reaction independent from the treatment [19].

\section{Conclusion}

JIA is a chronic disease of childhood period. The activetion of the disease and the medications used (steroid and DMARD combination) affect the PPD responses and cause difficulties in the evaluation of latent TB. PPD responses that are lower than cut-off values which are acceptable for the healthy children with and without vaccine can point out the underlying tuberculosis infection for the active JIA patients who are treated with methylprednisolone.

\section{REFERENCES}

[1] T. J. Cassidy and R. E. Patty, "Juvenile Rheumatoid Arthritis. Textbook of Pediatric Rheumatology," 3rd Edition, W. B. Saunders, 1995, pp. 133-223.

[2] R. N. Lipnick, G. C. Tsokov and D. B. Maglavy, "Immune Abnormalities in the Pathogenesis of Juvenile Rheumatoid Arthritis," Rheumatic Disease Clinics of North America, Vol. 17, 1991, pp. 703-859.

[3] D. Ponce de Leon, E. Acevedo-Vasquez, A. SanchezTorres, et al., "Attenuated Response to Purified Protein Derivative in Patients with Rheumatoid Arthritis: Study in a Population with a High Prevalence of Tuberculosis," Annals of the Rheumatic Diseases, Vol. 64, 2005, pp. 1360-1361. 
http://dx.doi.org/10.1136/ard.2004.029041

[4] Turkish Ministry of Health Division of Tuberculosis, "Incidence of Tuberculosis and Number of Cases over Years," 2000.

[5] American Academy of Pediatrics, "Report of the Committee on Infectious Disease. Red Book," 24th Edition, 1997, p. 544.

[6] F. Wolfe, K. Michaud, J. Anderson and K. Urbansky, "Tuberculosis Infection in Patients with Rheumatoid Arthritis and the Effect of Infliximab Therapy," Arthritis \& Rheumatism, Vol. 2, 2004, pp. 372-379. http://dx.doi.org/10.1002/art.20009

[7] H. Güvenç, A. Koç and O. Özkarakas, "PPD Skin Testing in Schoolchildren with and without BCG Vaccination," Turkish Journal of Medical Research, Vol. 11, No. 3, 1993, pp. 116-119.

[8] A. Koç and T. Karagöz, "Tüberkülozda Epidemiyolojik Ölçütler ve Hasta Grubu Analizi," Solunum Hastalıkları Dergisi, Vol. 8, No. 4, 1997, pp. 621-634.

[9] R. L. Sepulveda, X. Ferrer and R. U. Sorensen, "The Influence of Calmette Guerin Bacillus Immunization on the Booster Effect to Tuberculin Testing in Healthy Young Adults," The American Review of Respiratory Disease, Vol. 142, 1990, pp. 24-28. http://dx.doi.org/10.1164/ajrccm/142.1.24

[10] G. W. Comstock, L. B. Edwards and X. T. Nabang, "Tuberculin Sensitivity Eight to Fifteen Years after BCG Vaccination," The American Review of Respiratory Disease, Vol. 103, 1971, pp. 572-575.

[11] E. S. Uçan, C. Sevinç and Ö. Abadoglu, "Tüberkülin Testi Sonuçlarının Yorumlanması Ülkemiz Standartları ve yeni Gereksinimler,” Toraks Dergisi, Vol. 1, 2000, pp. 25-29.
[12] E. Bozkanat, F. Çiftci, M. Apaydın, Z. Kartaloglu, E. Tozkoparan, O. Deniz and O. Sezer, "Tuberculin Skin Test Screening in a Military School in Istanbul City Center."

[13] A. Sakar, T. Göktalay, L. Dagyıldızı and A. C. Yıldırım, "Manisa Ilinde Tüberküloz Taraması," Toraks Dergisi, 2001.

[14] J. Waxman, M. D. Lockshin, J. J. Schnapp and I. N. Doneson, "Cellular Immunity in Rheumatic Diseases I. Rheumatoid Arthritis," Arthritis \& Rheumatism, Vol. 4, 1974, pp. 499-506.

[15] H. M. Hoyeraal, "Impaired Delayed Hypersensitivity in Jüvenile Rheumatoid Arthritis," Annals of the Rheumatic Diseases, Vol. 32, 1973, p. 331. http://dx.doi.org/10.1136/ard.32.4.331

[16] J. R. Hayes, D. J. Ward and J. F. Jennings, "Studies on Cell-Mediated Hypersensitivity Responses in Rheumatoid Arthritis," Proceedings of the Third Symposium, 1970.

[17] M. G. Helliwell, G. S. Panayi and A. Unger, "Delayed Cutaneous Hypersensitivity in Rheumatoid Arthritis: The Influence of Nutrition and Drug Therapy," Clinical Rheumatology, Vol. 1, 1984, pp. 39-45. http://dx.doi.org/10.1007/BF02715694

[18] E. Kiray, O. Kasapcopur, V. Bas, et al., "Purified Protein Derivative Response in Juvenile Idiopathic Arthritis," Journal of Rheumatology, Vol. 36, 2009, pp. 2029-2032. http://dx.doi.org/10.3899/jrheum.090173

[19] I. Sezer, H. Kocabas, M. A. Melikoglu and M. Arman, "Positiveness of Purified Protein Derivatives in Rheumatoid Arthritis Patients Who Are Not Receiving Immunosuppressive Therapy," Clinical Rheumatology, Vol. 28, 2009, pp. 53-55. http://dx.doi.org/10.1007/s10067-008-0982-1

\author{
Abbreviations \\ JIA: Juvenile idiopathic arthritis \\ PPD: Purified Protein Derivative \\ RA: Rheumatoid Arthritis \\ ILAR: International League of Associations for Rheu- \\ matology \\ CHAQ: Childhood Health Assesment Questionnaire \\ DMARD: Disease Modifying Anti-rheumatic Drugs \\ TU: Turbidity Unit \\ BMI: Body Mass Index \\ BCG: Bacille Calmette-Guérin \\ TB: Tuberculosis
}

